\title{
Hijos de madres con enfermedad de Basedow Graves
}

\author{
Newborn of mothers with Graves' disease
}

\author{
Carola Goecke ${ }^{a}$, Francisca Grob ${ }^{a}$
}

aPontificia Universidad Católica de Chile, Facultad de Medicina, Escuela de Medicina, División de Pediatría. Santiago, Chile

Recibido el 26 de febrero de 2018; aceptado el 13 de agosto de 2018

\section{Resumen}

Introducción: La causa más frecuente de hipertiroidismo congénito es la enfermedad de Basedow Graves (EG) materna, en la que anticuerpos anti receptor de hormona tiroestimulante (TSH) (TRAb) atraviesan la placenta estimulando al receptor de TSH fetal y/o neonatal para producir hormonas tiroideas. La disfunción tiroidea en estos pacientes se confirma con el aumento de las concentraciones de tiroxina (T4) y triyodotironina (T3) acompañado de niveles de TSH suprimida. Objetivos: Caracterizar la evolución clínica y bioquímica de los recién nacidos (RN) hijos de madres con EG, y sugerir recomendaciones respecto al tratamiento y seguimiento. Material y Método: Se realizó una revisión de la literatura usando la base de datos MEDLINE, identificando artículos que incluyeran más de 30 $\mathrm{RN}$ de madres con EG y describieran su evolución. Se agregaron además revisiones del tema enfatizando la evaluación y manejo de los hijos de madres con EG. Resultados: Se incluyeron 9 estudios de cohorte que incorporaron 790 embarazadas. Hubo heterogeneidad entre los trabajos; un porcentaje variable de los hijos desarrolló tirotoxicosis neonatal, la que fue más frecuente cuando las madres presentaron concentraciones elevadas de TRAb. El tratamiento de los RN se inició según diferentes criterios. La literatura recomienda tratar los casos de hipertiroidismo clínico y considerarlo en casos de hipertiroidismo bioquímico. Conclusión: Los hijos de madres con EG y TRAb elevados deben ser evaluados por la probabilidad de desarrollar tirotoxicosis neonatal. Se sugiere controlar función tiroidea periódicamente durante el primer mes de vida y tratar los pacientes con hipertiroidismo clínico y bioquímico.
Palabras clave: Hipertiroidismo fetal; Hipertiroidismo neonatal; Enfermedad de Graves neonatal; Tirotoxicosis neonatal; Hijo de madre hipertiroidea

\section{Keywords:}

Fetal hyperthyroidism; Neonatal hyperthyroidism; Neonatal Grave's disease; Neonatal thyrotoxicosis; Neonates born to mothers with Graves 'disease 
these patients was included. Results: Nine cohort studies were included, with a total of 790 pregnant women. There was high heterogeneity among the studies. A variable percentage of newborns developed neonatal thyrotoxicosis, which was more frequent in those patients whose mothers had high levels of TRAb. The treatment of newborns was initiated according to different criteria. The literature recommends treating cases of clinical hyperthyroidism and considers it in cases of biochemical hyperthyroidism. Conclusion: Children of mothers with GD and high TRAb should be evaluated due to the likelihood of developing neonatal thyrotoxicosis. It is suggested to monitor thyroid function periodically during the first month of life and treat patients with clinical and biochemical hyperthyroidism.

\section{Introducción}

El hipertiroidismo congénito es una enfermedad poco frecuente, pero potencialmente severa. Su frecuencia es menor que la del hipotiroidismo congénito, pero su impacto en el crecimiento y el desarrollo puede ser tanto o más grave ${ }^{1}$. El exceso de hormonas tiroideas durante el periodo de recién nacido $(\mathrm{RN})$ puede interferir con el crecimiento y maduración cerebral, con secuelas neuro-anatómicas y en el neurodesarrollo ${ }^{2}$. Además, las hormonas tiroideas podrían aumentar la osteogénesis y de esta manera producir craneosinostosis.

La causa más frecuente de hipertiroidismo congénito es la enfermedad de Basedow Graves (EG) materna, en que anticuerpos anti receptor de hormona tiroestimulante (TSH) (TRAb) atraviesan la placenta y estimulan al receptor de TSH fetal y/o neonatal para producir hormonas tiroideas ${ }^{3}$. Estos anticuerpos pueden ser inmunoglobulinas del tipo estimulante o inhibitorio, y va a depender del ensayo que se utilice para determinarlos si es capaz de diferenciarlos o no ${ }^{4}$. Existen otras formas menos frecuentes de hipertiroidismo neonatal, causadas por variantes patogénicas activantes del receptor de TSH o del gen que codifica para la subunidad alfa de la proteína G (GNAS1), las que que inducen la activación constitutiva de cascadas de señalización intracelular, y en que los marcadores de autoinmunidad se encuentran negativos en el RN y su madre. Su presentación puede ser familiar y heredada de manera autosómica dominante, o producida por variantes patogénicas de novo ${ }^{5}$.

Además del riesgo de desarrollar hipertiroidismo, los hijos de madres con EG también pueden presentar hipotiroidismo, tanto primario como central. El hipotiroidismo primario, habitualmente es de curso transitorio, y se produce por una inhibición en la síntesis de hormonas tiroideas fetales y/o neonatales debido al paso transplacentario de las drogas antitiroideas usadas por la madre durante el embarazo o por el paso de otros anticuerpos antitiroideos inhibitorios además de los $\mathrm{TRAb}^{6}$. En casos de hipertiroidismo materno severo, el RN puede presentar un hipotiroidismo central, secundario a la inhibición del eje hipotálamo-hipofisario-tiroideo fetal/neonatal debido a concentraciones elevadas de hormonas tiroideas maternas circulantes que atraviesan la placenta e inhiben la síntesis de TRH y/o $\mathrm{TSH}^{7}$. Su incidencia se estima en 1:35.000 ${ }^{8}$, y al igual que el hipotiroidismo congénito clásico, puede producir daño al cerebro en desarrollo con grados variables de discapacidad intelectual.

El objetivo de este artículo es realizar una revisión de la literatura sobre la evolución clínica y bioquímica de los RN hijos de madres con EG, y sugerir recomendaciones de seguimiento y manejo de este grupo de pacientes.

\section{Material y Método}

Para la elaboración de esta revisión, un investigador en forma imparcial revisó la base de datos MEDLINE a través de PubMed, utilizando las palabras claves: "Hipertiroidismo neonatal", "Enfermedad de Graves neonatal", "Tirotoxicosis neonatal" e "Hijo de madre hipertiroidea" en idioma español e inglés; junto con los términos MESH "Graves Disease" e "Infant, Newborn".

Los criterios de inclusión fueron artículos científicos cuyo diseño fueran estudios de cohorte, publicados entre 1987 y 2017 (última búsqueda efectuada en Octubre 2017) en los que se caracterizara clínica y bioquímicamente a los RN hijos de madres con EG y que incluyeran más de treinta $\mathrm{RN}$ en el estudio. De éstos, se obtuvo la información materna, fetal y neonatal que estuviera disponible, incluyendo el uso de tratamiento farmacológico en caso de haberlo requerido. Se utilizó la lista de verificación PRISMA, como base para informar revisiones sistemáticas?.

La selección se basó en títulos y/o abstracts, y en la disponibilidad de artículos completos en idiomas español y/o inglés. Los artículos que no cumplieron los criterios mencionados fueron excluidos. Además, se revisaron las referencias de las publicaciones seleccionadas para identificar estudios relevantes no detectados por la búsqueda electrónica. Los datos de 
los artículos científicos se extrajeron en forma independiente.

No se recibió ningún tipo de financiamiento para la búsqueda de artículos científicos o para la elaboración de este manuscrito en general.

\section{Resultados}

Luego de la exclusión de una publicación por la escasez de datos reportados, se identificaron nueve artículos ${ }^{2,3,15-21}$ en los que se caracterizó a los hijos de madres con EG (Tabla 1).

De un total de $790 \mathrm{RN}$ de madres con antecedentes de EG reportados en nuestra búsqueda, un porcentaje variable de sus madres presentaron concentraciones de TRAb positivos durante el embarazo (18 a 42\%). De este grupo, entre 12 y $50 \%$ de los RN desarrollaron HTNN, es decir, entre un 2 y $21 \%$ de los hijos RN de todas las madres con EG desarrollaron HTNN. Sólo un estudio $^{3}$ determinó las concentraciones de TRAb tanto en las madres como en los RN. En éste, de $24 \mathrm{RN}$ que presentaron TRAb positivos en sangre de cordón, siete desarrollaron HTNN (29\%). La definición de hipertiroidismo neonatal varía de acuerdo a los diferentes autores, ya que puede establecerse de acuerdo a criterios bioquímicos (asintomáticos), clínicos, o ambos.

Dos artículos describieron la evolución de los RN con TRAb positivos, $15 \%$ y $29 \%$ desarrollaron HTTN respectivamente $e^{3,4}$. Por otro lado, cuatro artículos describieron la presentación clínica del HTNN en bioquímico (asintomático) ${ }^{4}$, clínico ${ }^{11}$, o ambos ${ }^{12,16}$. La mediana de TSH y tiroxina total/tiroxina libre (T4T/T4L) en los artículos que los reportaban fue de $0,71 \mathrm{mUI} / \mathrm{ml}$ y $22,7 \mathrm{ug} / \mathrm{dl} / 3,62 \mathrm{ng} / \mathrm{dl}$ en los casos de hipertiroidismo bioquímico, y de $0,59 \mathrm{mUI} / \mathrm{ml}$ y $29,2 \mathrm{ug} / \mathrm{dl} / 3,8 \mathrm{ng} / \mathrm{dl}$ en los casos de hipertiroidismo clínico respectivamente, aunque con distintos métodos de ensayo de laboratorio, por lo que en estricto rigor no serían comparables. Los síntomas de los recién nacidos fueron similares entre los trabajos, describiéndose inquietud, irritabilidad, mal incremento ponderal, sudoración, taquicardia, taquipnea, exoftalmo y bocio, destacando un caso de óbito fetal secundario a insuficiencia cardiaca congestiva por hipertiroidismo fetal ${ }^{12}$. La mayoría reportó uso de tratamiento médico (drogas antitiroideas o metimazol) frente a HTNN, ya sea bioquímico ${ }^{3}$, clínico ${ }^{10,15}$ o ambos ${ }^{4,14}$.

\section{Evaluación de la embarazada con enfermedad de Basedow Graves (EG)}

En todas las embarazadas con EG actual o en remisión (definida como posterior al tratamiento con drogas antitiroideas, radioyodo o cirugía), se deben determinar las concentraciones de TRAb. La Sociedad Americana de Tiroides (ATA) ${ }^{17}$ recomienda determinar TRAb al inicio del embarazo y luego a las 18 a 22 semanas. Si en este momento, las concentraciones de TRAb son elevadas o la madre mantiene los requerimientos de drogas antitiroideas, se deben volver a determinar entre las semanas 30 a 34 de embarazo. Si en cualquier momento los TRAb son negativos, el RN no requiere seguimiento específico ${ }^{18}$.

Una vez establecida la presencia de TRAb en la madre, es importante determinar su función tiroidea, ya que la tirotoxicosis materna se asocia a alteraciones neurocognitivas en su descendencia. Un estudio prospectivo ${ }^{19}$ que evaluó las concentraciones de hormonas tiroideas maternas durante las semanas 9 a 18 de gestación, y posteriormente, el coeficiente intelectual (CI) de sus hijos a una edad promedio de 6 años, demostró que tanto niveles altos como bajos de T4L reducían 1,4 a 3,8 puntos el CI del descendiente. Esta asociación se mantuvo incluso al excluir del análisis a las mujeres con hipo o hipertiroidismo clínico. Por este motivo, todos los hijos de madre con EG con TRAb positivos se encuentran en riesgo de presentar una disfunción tiroidea fetal desde la segunda mitad del embarazo, y a medida que aumenta la transferencia placentaria de TRAb, se debe determinar el estatus tiroideo de feto in útero a través de la evaluación de criterios maternos y fetales (Tabla 2) ${ }^{16}$. Los factores maternos que se asocian a disfunción tiroidea neonatal son: niveles elevados de T4 libre durante el embarazo, TRAb elevados durante el tercer trimestre 2 a 4 veces por sobre el límite superior de normalidad del ensayo ${ }^{11}$, uso de dosis altas de drogas antitiroideas y mayor tiempo de duración de hipertiroidismo durante el embarazo ${ }^{10}$. Los niveles de TRAb maternos durante el tercer trimestre del embarazo se correlacionan en forma perfecta con los TRAb de cordón umbilical. Un estudio ${ }^{20}$ demostró que concentraciones maternas de TRAb mayor a 2,5 UI/l predecían de mejor forma alteraciones de la función tiroidea fetal (sensibilidad $100 \%$, especificidad $64 \%$ ), y concentraciones de TRAb mayor a $6.8 \mathrm{UI} / \mathrm{L}$ en $\mathrm{RN}$ predijo alteraciones de la función tiroidea neonatal (sensibilidad $100 \%$, especificidad 94\%). En relación a la determinación de hormonas tiroideas de cordón umbilical, ésta no ha demostrado ser capaz de predecir la evolución de la función tiroidea neonatal, por lo que no se recomienda ${ }^{21}$.

\section{Evaluación del feto en riesgo de enfermedad de Graves}

Durante la evaluación de los fetos en riesgo de desarrollar tirotoxicosis, se debe monitorizar de cerca la 


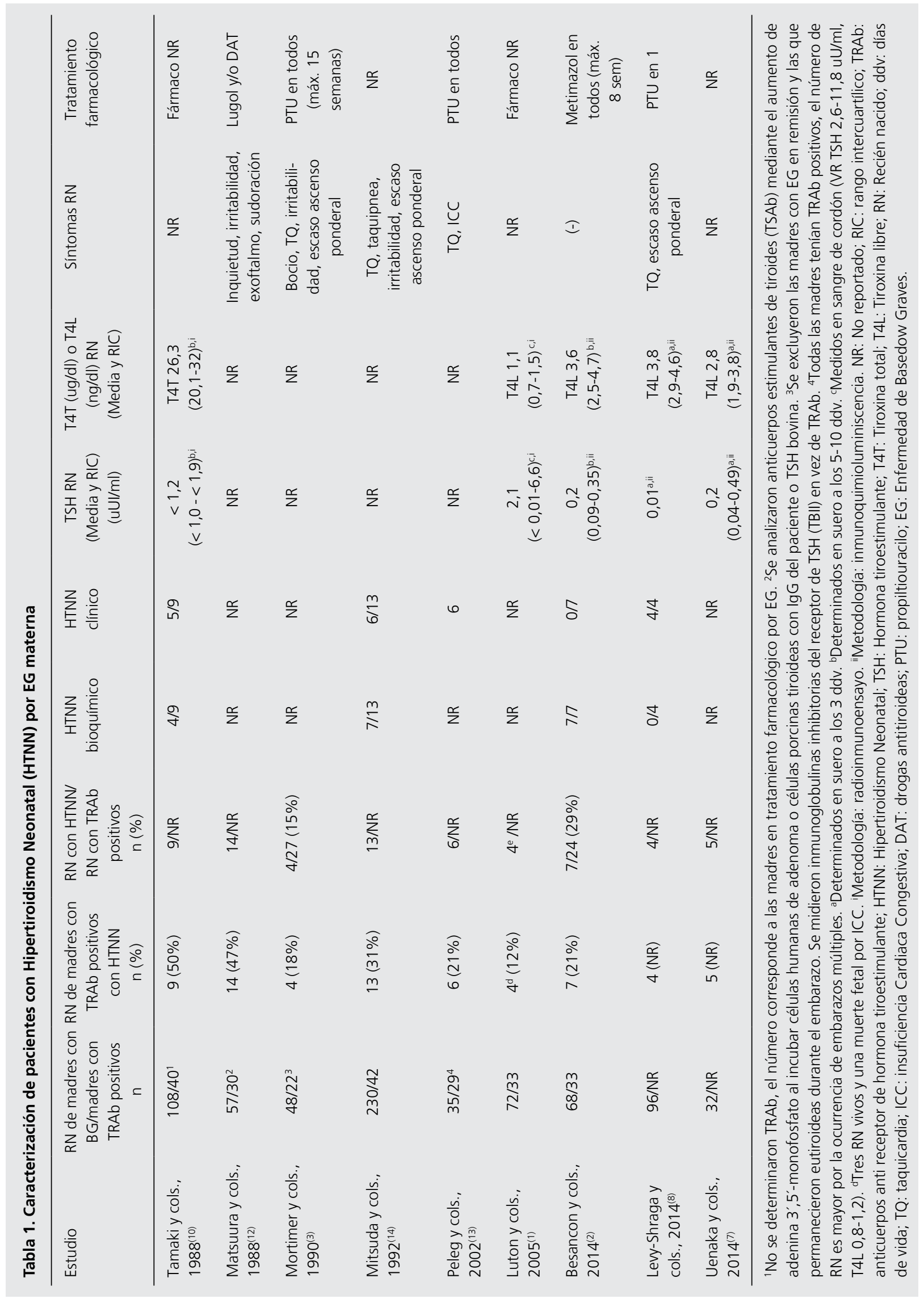


Enfermedad de Graves neonatal - C. Goecke et al

Tabla 2. Criterios maternos y fetales para sospecha de hipertiroidismo fetal

\begin{tabular}{|c|c|}
\hline Criterios maternos & Criterios fetales \\
\hline Niveles de TRAb elevados & Ecografía tiroidea: Tamaño y doppler tiroideo \\
\hline Drogas antitiroideas & Frecuencia cardíaca > 160 lpmb $^{b}$ \\
\hline Dosis máxima diaria & \\
\hline Dosis total utilizada & Edad ósea adelantadac \\
\hline Persistencia del hipertiroidismo durante el tercer trimestre de embarazo & Retraso del crecimiento intrauterino \\
\hline
\end{tabular}

aparición de bocio. Éste es el mejor signo de disfunción tiroidea fetal, y debe ser evaluado cuidadosamente a través de nomogramas tiroideos fetales basados en la edad gestacional y el diámetro biparietal ${ }^{22}$. Su presencia puede ser la manifestación tanto de un hipertiroidismo como de un hipotiroidismo: las manifestaciones clínicas dependerán del balance entre anticuerpos estimulantes y bloqueantes del receptor de $\mathrm{TSH}^{22}$. Los signos de tirotoxicosis fetal incluyen taquicardia, retraso de crecimiento intrauterino, adelanto de la edad ósea, insuficiencia cardíaca con hidrops no inmune, parto prematuro, y craneosinostosis ${ }^{23}$. La cordocentesis está raramente indicada para establecer la función tiroidea fetal $^{21}$, y sólo debe realizarse cuando se detecta bocio fetal en una mujer sin EG, si la función tiroidea fetal no puede inferirse por las características ecográficas del bocio y si es necesario el tratamiento con tiroxina intramniótica para revertir el polihidramnios progresivo $^{24}$. El hipertiroidismo fetal puede ser tratado en forma segura a través de la administración de drogas antitiroideas a la madre.

\section{Evaluación del $\mathrm{RN}$ en riesgo de enfermedad de Graves}

Posterior al nacimiento, todos los hijos de madres con EG con TRAb positivos o no determinados durante el embarazo, deben ser evaluados por el riesgo de desarrollar tirotoxicosis. Los signos clínicos de hipertiroidismo pueden aparecer algunos días después de nacer, cuando ya han desaparecido de la circulación las drogas anti tiroideas recibidas por la madre, si es que ésta fue tratada ${ }^{1}$. Hasta el $5 \%$ de los hijos de madre con EG puede desarrollar HTNN, por lo que es una patología infrecuente, cuya incidencia se estima en 1:25.000 a 1:50.000 ${ }^{23}$. Las manifestaciones clínicas pueden ser inespecíficas e inicialmente podrían atribuirse a infecciones virales congénitas o sepsis si no existe el antecedente de EG en la madre. Después del nacimien- to el RN puede presentar taquicardia, irritabilidad con temblores, dificultad para alimentarse, mal incremento ponderal, sudoración y dificultad para conciliar el sueño secundario a la tirotoxicosis. Pueden presentarse con un aspecto enflaquecido, proptosis con mirada fija, retracción palpebral y bocio. Otros signos menos frecuentes, que pueden ser interpretados como sepsis incluyen la trombocitopenia, hepatomegalia e ictericia $^{19}$.

\section{Hipertiroidismo neonatal}

El diagnóstico de hipertiroidismo neonatal se confirma con el aumento de las concentraciones de T4 y T3 acompañado de niveles de TSH suprimida. Se sugiere determinar concentraciones de TRAb apenas sea posible, ya sea en cordón o en sangre del RN, ya que esto permitirá dar de alta a los neonatos con anticuerpos negativos ${ }^{1,3}$. Los recién nacidos con TRAb positivos y función tiroidea normal durante los primeros días de vida deben mantenerse en seguimiento clínico y bioquímico por la posibilidad de hipertiroidismo de aparición tardía (figura 1). Las hormonas tiroideas deben interpretarse de acuerdo a los valores de referencia específicos para el periodo de $\mathrm{RN}$ y al método de laboratorio que se utilice, ya que posterior al peak de TSH neonatal durante las primeras horas de vida, se produce un aumento en las concentraciones de T4 que se mantiene durante los primeros 5 días de vida ${ }^{25}$.

El tratamiento del $\mathrm{RN}$ con drogas antitiroideas debe evaluarse caso a caso, ya que la evidencia no es concluyente en relación a que pacientes deben ser tra$\operatorname{tados}^{18}$. Existen pocos estudios, con números pequeños de pacientes, ausencia de grupos de control y falta de outcomes claros que permitan establecer criterios de selección de tratamiento. Por otra parte, existe escasa evidencia sobre los resultados a largo plazo, especialmente neurocognitivos, de los sujetos afectados con hipertiroidismo neonatal, tanto si fueron o no trata- 


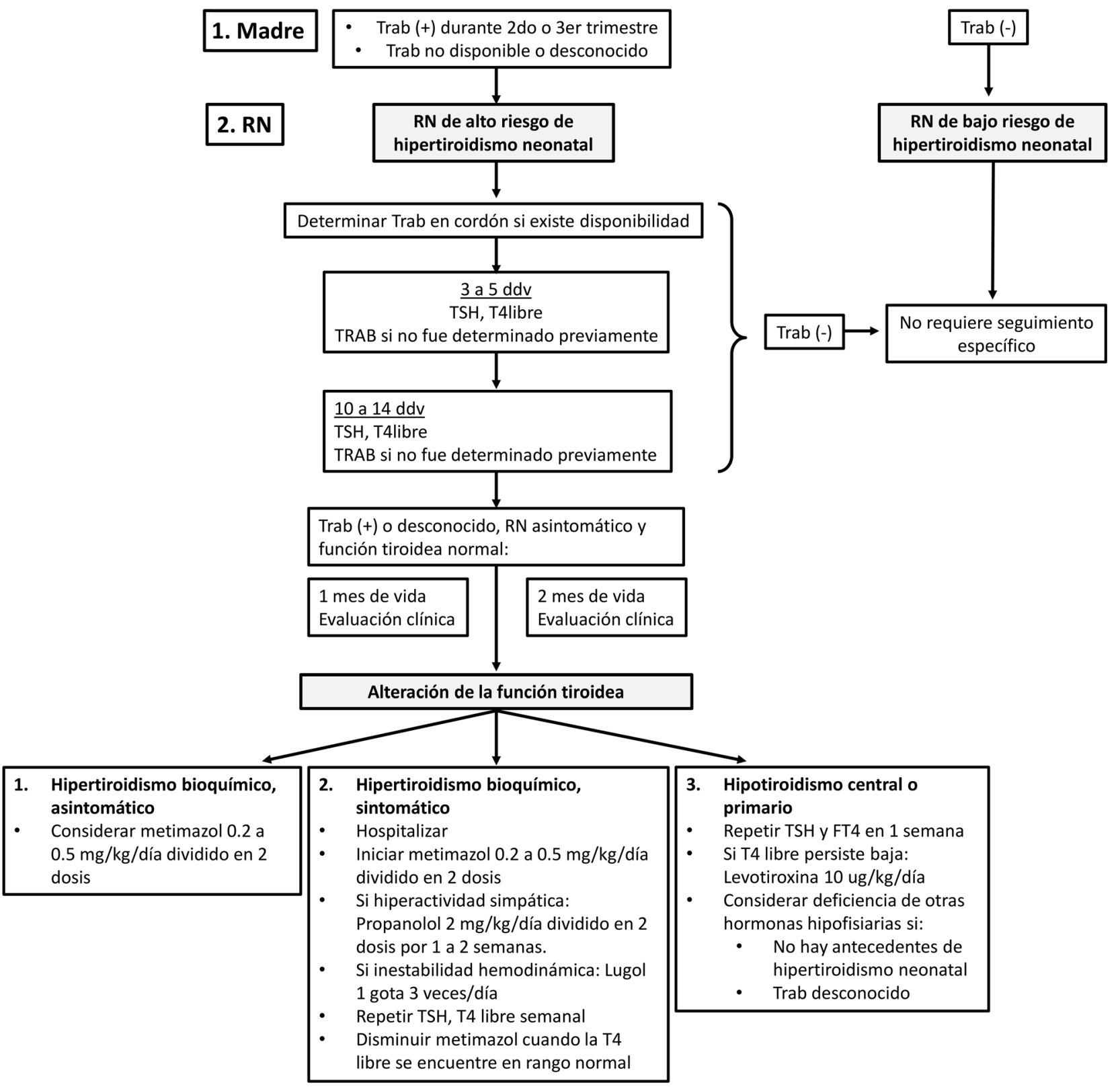

\section{RN de alto riesgo}

- Maternos

-TRAb (+) o desconocidos durante embarazo

-Tirotoxicosis clínica durante 3er trimestre

-Requerimientos de drogas antitiroideas durante 3er trimestre

-Historia familiar de mutación de TSHR

- Recién nacido

-Tirotoxicosis fetal

\section{RN de bajo riesgo}

- Maternos

-TRAb (-) durante 2do o 3er trimestre de embarazo

- Recién nacido

-TRAb (-) de cordón

Figura 1. Algoritmo de seguimiento del hijo de madre hipertiroidea. TSHR: Receptor de TSH. Adaptado de van der Kaay DC, Pediatrics 2016;137(4):e20151878. ddv = días de vida. 
dos. El objetivo del tratamiento con drogas antitiroideas es evitar la posibilidad de presentar consecuencias adversas relacionadas a la exposición a concentraciones elevadas de hormonas tiroideas, las que pueden presentarse a corto (insuficiencia cardíaca) o largo plazo (craneosinostosis, discapacidad cognitiva). Por lo tanto, frente a un recién nacido con síntomas y/o signos de hipertiroidismo asociado a hipertiroidismo bioquímico $^{18}$, se sugiere iniciar tratamiento con drogas antitiroideas. En éstos casos, se sugiere utilizar metimazol 0,2 a 0,5 mg kg/día dividido en dos dosis diarias $^{1}$. No se sugiere utilizar propiltiouracilo (PTU) por los potenciales efectos adversos graves. La respuesta puede no ser inmediata ya que este fármaco bloquea la síntesis, pero no la liberación de hormonas tiroideas. La función tiroidea neonatal debe ser monitorizada de cerca, ya que el hipotiroidismo inducido secundario al tratamiento con metimazol puede ser tanto o más perjudicial que el hipertiroidismo. Los beta bloqueadores son eficaces en el control de los síntomas adrenérgicos e inhiben la deyodación de T4 a T3. Se sugiere utilizar propranolol $2 \mathrm{mg} / \mathrm{kg} /$ día dividido en 2 dosis asociado al metimazol, monitorizando potenciales efectos adversos como hipoglicemia, bradicardia e hipotensión ${ }^{26}$. En RN tirotóxicos la solución de yodo que suprime la síntesis e inhibe la liberación de hormonas tiroideas, puede utilizarse en conjunto a las drogas antitiroideas (Lugol 1 gota 3 veces al día) y en casos graves se pueden agregar corticoides que disminuyen la conversión periférica de $\mathrm{T} 4$ a T3${ }^{18}$.

El HTNN secundario a EG materna es autolimitado y su duración dependerá de la velocidad de desaparición de los TRAb de la circulación del paciente. Se debe controlar la función tiroidea cada 1 a 2 semanas hasta la titulación de la dosis de metimazol y posteriormente cada 2 semanas ${ }^{18}$. Normalmente la duración total de la terapia es de 1 a 2 meses, y generalmente a los 6 meses ya se ha resuelto.

\section{Conclusiones}

Los trastornos tiroideos neonatales, tanto hipo e hipertiroidismo son enfermedades potencialmente graves que pueden producir discapacidad cognitiva severa e irreversible. Se analizaron 9 estudios de cohorte que describieran la evolución clínica y/o bioquímica de un número mayor de 30 hijos de madres con EG. Se observaron diferencias en la evaluación de las embarazadas y sus hijos. Un porcentaje variable de los $\mathrm{RN}$ desarrolló alteraciones clínicas y/o bioquímicas compatibles con hipertiroidismo, las que se describen con mayor frecuencia en los hijos de madres con concentraciones de TRAb muy elevadas durante el tercer trimestre del embarazo (27). Dado la heterogeneidad de los estudios, su interpretación es compleja y no permiten establecer criterios específicos de tratamiento. Por esta razón, el manejo de los hijos de madres con EG debe ser individualizado y monitorizado rigurosamente. En esta revisión, presentamos sugerencias para el estudio y tratamiento de la madre con EG y de su hijo(a) en riesgo de desarrollar una disfunción tiroidea.

\section{Conflicto de intereses}

Las autoras declaran no tener conflicto de intereses.

\section{Referencias}

1. Léger J, Olivieri A, Donaldson M, et al. European society for paediatric endocrinology consensus guidelines on screening, diagnosis, and management of congenital hypothyroidism. Horm Res Paediatr 2014; 81(2):80-103.

2. Daneman D, Howard NJ. Neonatal thyrotoxicosis: Intellectual impairment and craniosynostosis in later years. J Pediatr 1980; 97(2):257-9.

3. Besançon A, Beltrand J, Le Gac I, Luton D, Polak M. Management of neonates born to women with Graves' disease: A cohort study. Eur J Endocrinol 2014; 170(6):855-62.

4. Mortimer Rh, Tyack Sa, Galligan JP, Perry Keene Da, Tan Ym. Graves'disease In Pregnancy: Tsh Receptor Binding Inhibiting Immunoglobulins And Maternal And Neonatal Thyroid
Function. Clin Endocrinol (Oxf) 1990; 32(2):141-52.

5. Léger J. Management of Fetal and Neonatal Graves' Disease. Horm Res Paediatr 2017; 87(1):1-6.

6. Volumenie JL, Polak M, Guibourdenche J, et al. Management of fetal thyroid goitres: A report of 11 cases in a single perinatal unit. Prenat Diagn 2000; 20(10):799-806.

7. Grob F, Martínez-Aguayo A. Hipotiroidismo congénito: Un diagnóstico que no debemos olvidar. Rev Chil Pediatr 2012; 83(5):482-91.

8. Kempers MJ, van Tijn DA, van Trotsenburg AS, de Vijlder JJ, Wiedijk BM, Vulsma T. Central congenital hypothyroidism due to gestational hyperthyroidism: detection where prevention failed. J Clin Endocrinol Metab 2003; 88(12):5851-7.

9. Moher D, Liberati A, Tetzlaff J,
Altman DG; PRISMA Group. Preferred reporting items for systematic reviews and meta-analyses: The PRISMA statement. PLoS Med 2009; 6(7):e1000097.

10. Uenaka M, Tanimura K, Tairaku S, Morioka I, Ebina Y, Yamada H. Risk factors for neonatal thyroid dysfunction in pregnancies complicated by Graves' disease. Eur J Obstet Gynecol Reprod Biol 2014;177:89-93.

11. Levy-Shraga Y, Tamir-Hostovsky L, Boyko V, Lerner-Geva L, Pinhas-Hamiel O. Follow-Up of Newborns of Mothers with Graves' Disease. Thyroid 2014; 24(6):1032-9

12. Tamaki H, Amino N, Aozasa M, et al. Universal predictive criteria for neonatal overt thyrotoxicosis requiring treatment. Am J Perinatol 1988;5(2):152-8.

13. Luton D, Le Gac I, Vuillard E, et al. Management of Graves' disease during pregnancy: The key role of fetal thyroid 
gland monitoring. J Clin Endocrinol Metab. 2005; 90(11):6093-

14. Matsuura N, Fujieda K, Iida Y, et al. TshReceptor Antibodies In Mothers With Graves' Disease And Outcome In Their Offspring. Lancet 1988; 1:14-7.

15. Peleg D, Cada S, Peleg A, Ben-Ami M. The relationship between maternal serum thyroid-stimulating immunoglobulin and fetal and neonatal thyrotoxicosis. Obstet Gynecol 2002; 99(6):1040-3.

16. Mitsuda N, Tamaki H, Amino N, Hosono T, Miyai K, Tanizawa O. Risk factors for developmental disorders in infants born to women with Graves disease. Obstet Gynecol 1992; 80(3 Pt 1):359-64.

17. Alexander EK, Pearce EN, Brent GA, et al. 2017 Guidelines of the American Thyroid Association for the Diagnosis and Management of Thyroid Disease During Pregnancy and the Postpartum. Thyroid Am Thyroid Assoc 2017; 27(3):315-89.

18. van der Kaay DCM, Wasserman JD, Palmert MR. Management of Neonates Born to Mothers With Graves Disease. Pediatrics 2016; 137(4):e20151878-e20151878

19. Korevaar TI, Muetzel R, Medici M, et al.
Association of maternal thyroid function during early pregnancy with offspring IQ and brain morphology in childhood: a population-based prospective cohort study. Lancet Diabetes Endocrinol 2016; 4(1):35-43.

20. Banigé M, Estellat C, Biran V, et al. Study of the factors leading to foetal and neonatal dysthyroidism in children of patients with Graves' disease. J Endocr Soc 2017;1:751-61.

21. Williams FL, Simpson J, Delahunty C, et al. Developmental trends in cord and postpartum serum thyroid hormones in preterm infants. J Clin Endocrinol Metab 2004;89(11):5314-20.

22. Ranzini AC, Ananth CV., Smulian JC, Kung M, Limbachia A, Vintzileos AM. Ultrasonography of the fetal thyroid: Nomograms based on biparietal diameter and gestational age. J Ultrasound Med 2001; 20(6):613-7.

23. Polak M, Legac I, Vuillard E, Guibourdenche J, Castanet M, Luton D. Congenital hyperthyroidism: The fetus as a patient. Horm Res 2006; 65(5):235-42.

24. HYPERLINK "https://www.ncbi.nlm. nih.gov/m/pubmed/?term=Stoppa-
Vaucher\%20S\%5BAuthor\%5D \&sort=ac\&from=/21745105/ac" Stoppa-Vaucher S, HYPERLINK "https://www.ncbi.nlm.nih.gov/m/ pubmed/?term=Van\%20Vliet\%20G\%5B Author\%5D\&sort=ac\&from $=/ 21745105 /$ ac" Van Vliet G, HYPERLINK "https://www.ncbi.nlm.nih.gov/m/ pubmed/?term=Delado\%C3\%ABy\%20 J\%5BAuthor\%5D\&sort=ac\&fr om=/21745105/ac" Deladoëy J. Discovery of a fetal goiter on prenatal ultrasound in women treated for Graves' disease: first, do no harm. Thyroid. 2011; 21(8):931.

25. Chaler EA, Florenzano R, Chilelli C, et al. Age-specific thyroid hormone and thyrotropin reference intervals for a pediatric and adolescent population. Clin Chem Lab Med. 2012;50(5):885-90.

26. Grüters A, Biebermann H, Krude H. Neonatal thyroid disorders. Horm Res 2003; 59(Suppl. 1):24-9.

27. van Dijk MM, Smits IH, Fliers E, Bisschop PH. Maternal Thyrotropin Receptor Antibody Concentration and the Risk of Fetal and Neonatal Thyrotoxicosis: A Systematic Review. Thyroid 2018; 28(2):257-64. 